\title{
AÇÃO EXTENSIONISTA DE PROMOÇÃO DA SAÚDE E PREVENÇÃO DE DOENÇAS CARDIOVASCULARES PARA ALUNOS DE UMA ESCOLA DA BAHIA
}

\author{
Aline Prates Correia \\ Universidade Federal do Sul da Bahia \\ aline_prates2007@hotmail.com \\ Fábio Jambeiro Santana Borges \\ Universidade Federal do Sul da Bahia \\ fabiojambeiroborges@gmail.com \\ Karolina de Oliveira Lima \\ Universidade Federal do Sul da Bahia \\ karol.lima22@hotmail.com
}

Kawan Moreira Santana Universidade Federal do Sul da Bahia kawankms@gmail.com

Laila Cheibub Costa Rodrigues Universidade Federal do Sul da Bahia lailacheibub@yahoo.com.br

Grasiely Faccin Borges Universidade Federal do Sul da Bahia grasiely.borges@gmail.com

\section{Resumo}

Objetivo: Relatar a experiência de uma ação educativa sobre prevenção de doenças relacionadas aos hábitos de vida realizada em uma escola no Extremo Sul da Bahia. Método: Foram realizados 3 encontros de 4 horas, com 76 estudantes do nono ano. As sessões discutiram temas relacionados a medidas antropométricas, cardiometabólicas e nível de atividade física, a partir das informações dos estudantes, evidenciando a importância da prevenção de doenças. Resultados: Participaram ativamente 62 estudantes, com idade média de 14,74 $\pm 0,95$ anos. Os adolescentes associaram as informações de índice de massa corporal, relação cintura e quadril, pressão arterial sistólica e diastólica, nível de atividade física com as doenças relacionadas ao estilo de vida de cada encontro. Conclusão: Pode-se inferir que a ação contribuiu com o autoconhecimento dos estudantes sobre melhores hábitos de vida e prevenção de doenças.

Palavras-chave: Relações Comunidade-Instituição. Estilo de Vida. Doenças Cardiovasculares.

\section{EXTENSIONIST ACTION TO PROMOTE HEALTH AND PREVENTION OF CARDIOVASCULAR DISEASES FOR STUDENTS AT A SCHOOL IN BAHIA}

\begin{abstract}
Objective: Report the experience of educational activity on the prevention of diseases related to lifestyle habits carried out in a school in the Far South of Bahia. Method: Three meetings lasting 4 hours were held with 76 students, of ninth grade. The sessions discussed topics related to anthropometric, cardiometabolic, and physical activity measures, based on information from students, highlighting the importance of disease prevention. Results: 62 students actively participated, with a mean age of $14.74 \pm 0.95$ years. Adolescents associated information on body mass index, waist and hip ratio, systolic and diastolic blood pressure, level of physical activity with diseases related to the lifestyle of each meeting. Conclusion: It can be inferred that the action contributed to the students' self-knowledge about better lifestyle habits and disease prevention.

Keywords: Community-Institutional Relations. Life Style. Cardiovascular Diseases.

\section{ACCIÓN EXTENSIONISTA PARA PROMOVER LA SALUD Y LA PREVENCIÓN DE ENFERMEDADES CARDIOVASCULARES PARA ESTUDIANTES DE UNA ESCUELA EN BAHÍA}

\section{Resumen}

Objetivo: Informar la experiencia de una acción educativa sobre prevención de enfermedades relacionadas con hábitos de vida realizada en una escuela del extremo sur de Bahía. Método: Se realizaron tres reuniones de 4 horas con 76 alumnos de noveno grado. Las sesiones abordaron temas relacionados con las medidas antropométricas, cardiometabólicas y de actividad física, a partir de la información de los estudiantes, destacando la importancia de la prevención de enfermedades. Resultados: participaron activamente 62 estudiantes, con una edad media de 14,74 \pm 0,95 años. Los adolescentes asociaron información sobre índice de masa corporal, índice cintura y cadera, presión arterial sistólica y diastólica, nivel de actividad física con enfermedades relacionadas con el estilo de vida de cada encuentro. Conclusión: Se puede inferir que la acción contribuyó al autoconocimiento de los estudiantes sobre mejores hábitos de vida y prevención de enfermedades.

Palabras clave: Relaciones Comunidad-Institución. Estilo de Vida. Enfermedades Cardiovasculares. 
Ação extensionista de promoção da saúde e prevenção de doenças cardiovasculares para alunos de uma escola da Bahia

\section{INTRODUÇÃO}

A doença cardiovascular (DCV) é um problema de saúde crescente no mundo contemporâneo que afeta todas as idades. Embora a taxa de mortalidade venha decrescendo com o avanço da assistência médica à saúde, sua prevalência está aumentando, pois o sedentarismo, a má alimentação e outros fatores de risco estão presentes no cotidiano da vida moderna. Em decorrência desses maus hábitos de vida, as doenças metabólicas clássicas, como obesidade, dislipidemias, diabetes e hipertensão arterial, também contribuem para o maior risco de eventos cardiovasculares (PRÉCOMA et al., 2019; BORIM et al., 2020).

A saúde infanto-juvenil vem sendo alvo de preocupação diante do aumento do sobrepeso na infância, além do sedentarismo e má alimentação (CARVALHO, BELÉM, ODA, 2017). A implementação de políticas de saúde preventiva, que estimule hábitos de vida mais saudáveis é essencial para o controle das DCV em qualquer faixa etária (PRÉCOMA et al., 2019). A inatividade física, por exemplo, prejudica o desenvolvimento osteomuscular, os quais contribuem ativamente para manutenção e proteção do sistema cardiovascular. Quando aliado à má alimentação, aumenta sua predisposição ao desenvolvimento de doenças crônicas com o avançar da idade (MARINHO e RIBEIRO, 2019).

A abordagem atual sobre o processo saúde-doença visa prevenir o fluxo cultural e social que dão manutenção aos maus hábitos de vida (MADEIRA et al., 2018). Nesse sentido, a ação de extensão universitária se configura como uma importante ferramenta de promoção da saúde na comunidade, interpretando um papel educativo, na construção de novos hábitos de saúde para adolescentes do ensino fundamental (GONÇALVES et al., 2016). Além disso, também se considera o retorno educativo para os extensionistas que realizam essas ações, através do contato com a realidade (RABELO et al., 2016).

O presente trabalho descreve as ações extensionistas/educativas realizadas com adolescentes do ensino fundamental II, em uma escola de Teixeira de Freitas/BA, com vistas à prevenção de doenças e apresentação de um novo olhar sobre a saúde pessoal. Para contribuir com a intervenção, também foram oferecidas as análises de suas medidas antropométricas e cardiometabólicas, como instrumento de autoconhecimento.

\section{MATERIAIS E MÉTODOS}

Este relato de experiência foi aprovado pelo Edital da Pró-Reitoria de Sustentabilidade e Integração Social (PROSIS) no 14/2019, com caráter de extensão, realizado por dez estudantes do curso de medicina da Universidade Federal do Sul da Bahia (UFSB). 
Ação extensionista de promoção da saúde e prevenção de doenças cardiovasculares para alunos de uma escola da Bahia

A ação aconteceu na Escola Municipal Igualdade e Justiça, localizada no bairro carente Vila Vargas, em Teixeira de Freitas-BA. Segundo o IBGE (2021), essa cidade possui 162.438 habitantes com 0,685 na escala do índice de desenvolvimento humano (IDH), e ainda, uma rede municipal de educação que conta com 63 escolas. Anualmente, são matriculadas crianças e adolescentes, maioria de baixa renda, que possuem uma aula de educação física na semana, com duração de 40 minutos, e algumas vão para a escola caminhando ou de bicicleta. Seus hábitos alimentares são pobres em fibras e ricos em carboidratos e açúcares.

A ação extensionista aconteceu no período de outubro a dezembro de 2019. Inicialmente houve uma reunião com a diretora da escola para planejamento dos encontros, e, assim, foram definidos três momentos com as crianças e seus professores, um por semana consecutiva, cada um com duração de 4 horas. Destes encontros, participaram 76 estudantes de duas turmas de ensino fundamental II, no período do turno matutino, que era a turma com maior necessidade de atividades extracurriculares na escola. Essa intervenção de promoção de saúde contou com o auxílio de colaboradores da escola quanto à disponibilidade dos espaços de sala de aula para execução da ação. Além disso, os critérios de inclusão para participar da ação foram: estudantes do nono ano do Ensino Fundamental II, independente de sexo e etnia e que frequentaram os encontros nas datas pré-agendadas na escola.

No primeiro momento, foi solicitada a anuência de participação para os pais ou responsáveis das crianças pelo diretor da escola. Em seguida, foi obtida a assinatura do Termo de Assentimento Livre e Esclarecido (TALE), para que fossem obtidas informações antropométricas que foram utilizadas na discussão e repassados para os alunos como um retorno da ação de extensão, sobre a saúde e hábitos de vida de cada um.

Para o segundo momento da vivência foram organizados dois espaços: o primeiro para aferição da pressão arterial sistólica e diastólica, e medição da frequência cardíaca (FC) (BARROSO et al., 2020). O segundo espaço foi preparado para a medida dos dados antropométricos dos estudantes, como estatura, massa corporal, circunferência da cintura (CC) e circunferência do quadril (CQ). Após isso, cada criança era orientada em uma roda de conversa sobre a importância de atualizar a rotina de acompanhamento hemodinâmico e antropométrico como prevenção de doenças.

No terceiro momento, foi estruturado um ambiente para a aferição da glicemia capilar aleatória, cumprindo os cuidados necessários quanto ao protocolo de aferição e higienização. Para o diagnóstico de diabetes, a Sociedade Brasileira de Diabetes considera a medida de glicose ao acaso de $\geq 200 \mathrm{mg} / \mathrm{dL}$ associado a sintomas de hiperglicemia (poliúria, polidipsia, polifagia e perda de peso) (SOCIEDADE BRASILEIRA DE DIABETES, 2019). Os alunos participaram 
Ação extensionista de promoção da saúde e prevenção de doenças cardiovasculares para alunos de uma escola da Bahia

da roda de conversa sobre doenças metabólicas nessa etapa, para tirar dúvidas e compreender como funciona a evolução clínica do Diabetes Mellitus 1 e 2.

No quarto momento da vivência, foi organizado um terceiro espaço para a aplicação do International Physical Activity Questionnaire (IPAQ), na versão curta. Esse momento foi essencial para garantir o entendimento das crianças sobre quanto tempo de atividade física deveriam fazer para prevenir o sedentarismo, e estimular uma vida mais ativa. Em todos os encontros foi disponibilizado um espaço de discussão a respeito dessas informações, com esclarecimento de dúvidas sobre os temas. No último encontro, as crianças foram reunidas e fizemos uma roda de conversa sobre hábitos de vida, e a importância da prática de exercícios físicos para a prevenção de doenças.

A identificação e classificação de risco de obesidade, que foi realizada no segundo momento, para garantir o autoconhecimento dos jovens sobre saúde, foi obtido o Índice de Massa Corporal (IMC) que classifica o excesso de peso corporal em: sobrepeso, obesidade I, obesidade II e obesidade III; e a relação cintura quadril (RCQ) que indica risco para doença cardiovascular. Após isso, foi possível relacionar o IMC e a RCQ, e identificar o risco de DCV nos alunos (MORAIS, 2019).

O IMC é feito pela divisão do peso pelo quadrado de sua altura, e classificado com os parâmetros adotados pela Organização Mundial da Saúde (OMS): baixo peso (IMC menor que $18,5 \mathrm{Kg} / \mathrm{m}^{2}$ ); peso ideal (IMC entre 18,5 e 24,9 Kg/m²); sobrepeso (IMC entre 25,0 e $29,9 \mathrm{Kg} / \mathrm{m}^{2}$ ); e obesidade (IMC maior ou igual a $30,0 \mathrm{~kg} / \mathrm{m}^{2}$ ). O RCQ foi obtido pela divisão das medidas de CC e CQ. O valor de referência de CC para o sexo feminino é menor que $80 \mathrm{~cm} \mathrm{e}$, para o sexo masculino, menor que $94 \mathrm{~cm}$; o valor de RCQ para o sexo feminino deve ser menor que 0,85 e para o sexo masculino menor que 0,90 (ASSOCIAÇÃO BRASILEIRA PARA O ESTUDO DA OBESIDADE E DA SÍNDROME METABÓLICA (ABESO), 2016).

Para discutir com os alunos sobre as informações referentes ao nível de atividade física, as interpretações foram de acordo com o critério baseado nas recomendações do IPAQ, em que os estudantes foram classificados em Ativo, Irregularmente Ativo e Sedentário, de acordo com a frequência e a duração das atividades físicas semanais, as quais, se inferiores a 150 minutos por semana, faz o estudante ser considerado irregularmente ativo, contribuindo para aumentar os riscos de desenvolver doenças (SILVA et al., 2007). As informações gerais sobre os alunos foram passadas para a escola e os estudantes, através da entrega de fichas individuais, para garantir o sigilo do nível de atividade física, seus dados antropométricos e cardiometabólicos, como forma de estímulo à busca de hábitos de vida saudáveis. 
Ação extensionista de promoção da saúde e prevenção de doenças cardiovasculares para alunos de uma escola da Bahia

\section{RESULTADOS E ANÁLISES}

A ação extensionista contou com a participação dos estudantes, educadores e coordenadores da escola, que participaram das discussões, compartilhando suas dúvidas e histórias relacionadas à prevenção de doenças e a promoção da saúde. Houve uma troca de saberes sobre temáticas do estilo de vida, como a hipertensão arterial, obesidade, sedentarismo, patologias osteomusculares e alimentação inadequada, como fatores causais de muitas doenças.

O projeto de extensão foi realizado com 76 estudantes. Destes, 14 foram excluídos pela descontinuidade da participação durante a ação. Dos 62 participantes, 41 eram do gênero masculino e 21 do gênero feminino, com idade média de 14,74 $\pm 0,95$ anos. Essa faixa etária é um alvo importante de mudanças, uma vez que estão em uma etapa mais suscetível às alterações de hábitos de vida. Além disso, apresentam o perfil de serem multiplicadores do conhecimento aprendido, o que enfatiza a necessidade da abordagem junto aos mesmos (RODRIGUES et.al., 2016).

Todos os processos foram realizados junto com os estudantes, que na etapa do IMC demonstraram ter dúvidas de como utilizar essa ferramenta e, principalmente, sobre sua aplicabilidade, o que, mais uma vez, denota a necessidade de trabalhar sobre o tema. A média do IMC foi de 21,61 $\pm 3,86 \mathrm{~kg} / \mathrm{cm}^{2}$, no qual 10 estudantes estavam com baixo peso, 42 com peso ideal, sete estavam com sobrepeso, dois com Obesidade grau I e um com Obesidade grau II. O perfil de IMC dos alunos diverge de outro estudo feito com estudantes adolescentes, no qual 44\% da amostra analisada apresentava excesso de peso (SALAZAR RENDÓN, MÉNDEZ, AZCORRA, 2018). Apesar de 42 estudantes apresentarem o peso ideal, 10 foram identificados com excesso de peso, questão que merece atenção devido ao risco de doenças crônicas (MATJUDA et al., 2020), pois a obesidade, as doenças cardiovasculares e os distúrbios metabólicos são problemas cada vez mais comuns em adolescentes (KOLMAGA, TRAFALSKA, SZATKO, 2019).

A relação cintura-quadril (RCQ) feminina teve média de $0,75 \pm 0,06 \mathrm{~cm}$ e a média masculina foi $0,78 \pm 0,06 \mathrm{~cm}$. Analisando os dois gêneros em conjunto, identificamos que 36 estudantes estavam com baixo risco, 15 risco moderado, oito alto risco e três com muito alto risco de DCV. Os estudantes não imaginavam sua realidade em saúde e foi enaltecida a necessidade de acompanhamento regular, uma vez que a circunferência da cintura (CC) serve como um índice importante para refletir a adiposidade central e é considerada mais fortemente associada a certos fatores de risco de doença cardiovascular em comparação ao IMC (ASSOCIAÇÃO 
Ação extensionista de promoção da saúde e prevenção de doenças cardiovasculares para alunos de uma escola da Bahia

BRASILEIRA PARA O ESTUDO DA OBESIDADE E DA SÍNDROME METABÓLICA (ABESO), 2016).

Neste estudo o gênero feminino compõe o maior quantitativo de indivíduos que apresentaram risco de DCV moderado $(\mathrm{n}=9)$ e, também, risco alto $(\mathrm{n}=5)$ e risco muito alto $(\mathrm{n}=3)$. As mulheres podem ter até 35 vezes mais chances de ter obesidade abdominal do que homens. Essa diferença na prevalência de obesidade abdominal entre mulheres e homens tem sido atribuída a vários fatores, incluindo: a diferença de hormônios sexuais que causam modificações na estrutura e na composição corporal, principalmente durante a adolescência, e também, diferenças entre o meio ambiente e a suscetibilidade genética do acúmulo de gordura entre ambos os gêneros (MALIK et al., 2019).

A prevenção de doenças ateroscleróticas, tem alto impacto sob a diminuição do risco de doenças cardiovasculares, por isso, faz-se necessário o acompanhamento dessas alunas, para que possam aprender mais sobre a temática e para evitar outros fatores de riscos para DCV, como o diabetes, tabagismo e hipertensão arterial (FALUDI et.al, 2017).

$\mathrm{Na}$ medida da pressão arterial observou-se média de PAS 120,25 $\pm 14,06 \mathrm{mmHg}$ e PAD

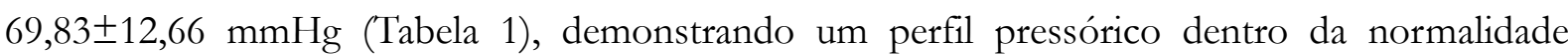
segundo a $7^{a}$ Diretriz Brasileira de Hipertensão arterial (MATJUDA et al., 2020). Apesar da maioria dos adolescentes apresentarem variáveis hemodinâmicas normais, é importante mencionar que a ligação entre obesidade abdominal e pressão alta está bem estabelecida (MALIK et al., 2019). Em relação à FC os dados revelaram valores dentro da normalidade com média de $82,40 \pm 12,31$ bpm (Tabela 1$)$.

A média da glicemia dos alunos foi de 95,19 $\pm 10,82 \mathrm{mg} / \mathrm{dL}$ (Tabela 1) e não houve medida maior ou igual a $200 \mathrm{mg} / \mathrm{dL}$. Apesar dos valores da glicemia estarem dentro da normalidade, os alunos relataram um alto consumo de açúcares e carboidratos, o que aumenta o risco de desenvolver diabetes, e ainda, a adolescência é um período de risco para o aparecimento de resistência à insulina devido à redução fisiológica na sensibilidade à mesma, causado pelo aumento dos hormônios sexuais e do hormônio do crescimento, responsáveis pelas mudanças físicas dessa fase do desenvolvimento (BARRACLOUGH et al., 2019). Assim sendo, faz-se importante a realização de ações no setor educacional, que priorizem a população de crianças, adolescentes e adultos jovens com a execução de ações orientadas para prevenção, detecção e controle do diabetes de forma precoce (SOCIEDADE BRASILEIRA DE DIABETES, 2019). 
Ação extensionista de promoção da saúde e prevenção de doenças cardiovasculares para alunos de uma escola da Bahia

Tabela 1 - Variáveis antropométricas, cardiovasculares e metabólicos de estudantes de Ensino Fundamental II (n=62) participantes do projeto de extensão.

\begin{tabular}{|c|c|}
\hline Variáveis & Média/Desvio Padrão \\
\hline Pressão Arterial Sistólica & $120,25 \pm 14,06 \mathrm{mmHg}$ \\
\hline Pressão Arterial Diastólica & $69,83 \pm 12,66 \mathrm{mmHg}$ \\
\hline Frequência Cardíaca & $82,40 \pm 12,31 \mathrm{bpm}$ \\
\hline Relação Cintura Quadril Feminino & $0,75 \pm 0,06 \mathrm{~cm}$ \\
Relação Cintura Quadril Masculino & $0,78 \pm 0,06 \mathrm{~cm}$ \\
\hline Índice de Massa Corporal & $21,61 \pm 3,86 \mathrm{~cm}$ \\
\hline Glicemia ao acaso & $95,19 \pm 10,82 \mathrm{mg} / \mathrm{dL}$ \\
\hline
\end{tabular}

Fonte: Autores, 2019.

O IPAQ demonstrou um total de atividades semanal de 785,04 $\pm 806,81$ min e tempo total sentado por final de semana de $958,87 \pm 434,18$ min. O estudo revelou que os escolares tinham hábitos que os levam a ser classificados como ativos. Contudo, os mesmos obtiveram um tempo em atividade sedentária maior que o tempo gasto em atividade física. Um estudo realizado com estudantes do ensino médio demonstrou que 50,83\% das pessoas não sabiam o que é sedentarismo e preconiza a importância de haver ensino e promoção de hábitos em exercício físico regular nas escolas (SANTOS; SERRA; PEREIRA, 2020).

A participação dos professores na atividade de intervenção possibilitou à escola autonomia para continuarem falando sobre prevenção e hábitos de vida saudáveis em meio aos componentes curriculares, promovendo, assim, uma educação em saúde continuada. As ações de extensão universitária são promotoras de interações transformadoras, por meio do estímulo para o desenvolvimento social e o raciocínio crítico dos estudantes participantes da ação, contribuindo com o aprendizado com o autocuidado e, consequentemente, com a melhoria da qualidade de vida (RABELO et al., 2016; FONSECA et al., 2020).

\section{CONSIDERAÇÕES FINAIS}

A ação extensionista trousse uma experiência relevante e contribuiu para firmar a importância do exercício da educação em saúde entre universidade e escola, pois o entendimento sobre autocuidado ocorreu através da troca de experiências entre os envolvidos. Nesse processo foi possível compreender a realidade dos estudantes, e sanar suas dúvidas e dificuldades, junto com eles com relação aos hábitos para um estilo de vida saudável.

Certas práticas de atividade física, como fazer caminhada ou andar de bicicleta, já eram realizadas por alguns estudantes, no entanto, eles não as consideravam como atividades de promoção do autocuidado. A falta de profundidade acerca desses assuntos do cotidiano foi aproveitada como conteúdo nas discussões, para melhor compreensão sobre os temas. 
Ação extensionista de promoção da saúde e prevenção de doenças cardiovasculares para alunos de uma escola da Bahia

Ampliamos a visão para as formas de avaliar e acompanhar o estado de saúde individual, com especial destaque para bons hábitos alimentares.

Pode-se inferir que a maior parcela dos jovens apresentou índices cardiometabólicos dentro do esperado, de acordo os parâmetros da Organização Mundial da Saúde e da Sociedade Brasileira de Cardiologia. A outra parcela, apesar de pertencerem a um grupo de risco, passaram a compreender sua situação e se mostraram interessados em continuar adquirindo conhecimento sobre autocuidado.

Dessa forma, as estratégias de promoção de saúde devem estar presentes na rotina das escolas com maior frequência, afinal, para mudar hábitos é importante que elas façam parte de uma prática rotineira. Isso enfatiza a importância de ações educativas em prevenção primária para os diversos segmentos que ocupam o espaço escolar. Assim, este trabalho proporcionou promoção de saúde e desempenhou importante função social, ao contribuir com um público em idade escolar.

\section{REFERÊNCIAS}

Associação Brasileira para o Estudo da Obesidade e da Síndrome Metabólica (ABESO). Diretrizes brasileiras de obesidade 2016 / ABESO - Associação Brasileira para o Estudo da Obesidade e da Síndrome Metabólica. - 4.ed. -São Paulo, SP.

BARRACLOUGH, J. Y. et al. Weight gain trajectories from birth to adolescence and cardiometabolic status in adolescence. The Journal of pediatrics, v. 208, p. 89-95. e4, 2019. https://doi.org/10.1016/j.jpeds.2018.12.034.

BARROSO, W. K. S., et al. Diretrizes Brasileiras de Hipertensão Arterial - 2020. Arquivo Brasileira de Cardiologia, v.116, n.3, p:516-658, 2021. https://doi.org/10.36660/abc.20201238.

BORIM, M. L. C. et al. Life habits, overweight and obesity of a group of children in training for running. Brazilian Journal of Development, v.6, n.4, p.19162-19174, apr. 2020. https://doi.org/10.34117/bjdv6n4-182.

CARVALHO, A. R. M.; BELÉM, M. D. O; ODA, J. Y. Sobrepeso e obesidade em alunos de 6-10 anos de escola estadual de Umuarama/PR. Arquivo de Ciência e Saúde da UNIPAR, $\begin{array}{lllll}\text { Umuarama/PR, } & \text { v.21, } & \text { n.1, } & \text { p.3-12, } & \text { jan./abr., }\end{array}$ https://doi.org/10.25110/arqsaude.v21i1.2017.6070.

FALUDI, A. A. et al. Atualização da diretriz brasileira de dislipidemias e prevenção da aterosclerose-2017. Arquivos brasileiros de cardiologia, v. 109, p. 1-76, 2017. https://doi.org/10.5935/abc.20170121.

FONSECA, Y. S. et al. Ação extensionista de promoção da saúde de estudantes universitários. Extensio: Revista Eletrônica de Extensão, v. 17, n. 35, p. 81-95, 2020. https://doi.org/ 10.5007/1807-0221.2020v17n35p81. 
Ação extensionista de promoção da saúde e prevenção de doenças cardiovasculares para alunos de uma escola da Bahia

GONÇALVES, L. F. F. et al. Promoção de saúde com adolescentes em ambiente escolar: relato de experiência. SANARE- Revista de Políticas Públicas, v.15 n.2, p.160-167, 2016.

IBGE - INSTITUTO BRASILEIRO DE GEOGRAFIA E ESTATÍSTICA. Censo Brasileiro de 2020. Rio de Janeiro: IBGE, 2020.

KOLMAGA, A; TRAFALSKA, E.; SZATKO, F. Risk factors of excessive body mass in children and adolescents in Lódz. Roczniki Państwowego Zakładu Higieny, v. 70, n. 4, 2019. https://dx.doi.org/10.32394/rpzh.2019.0085.

MADEIRA, F. B. et al. Estilos de vida, habitus e promoção da saúde: algumas aproximações. Saúde e Sociedade. 2018, v. 27, n. 1, pp. 106-115, 2018. https://doi.org/10.1590/S010412902018170520.

MALIK, S. K. et al. Prevalence of abdominal obesity and its correlates among adults in a periurban population of West Africa. AIMS public health, v. 6, n. 3, p. 334, 2019. https://dx.doi.org/10.3934\%2Fpublichealth.2019.3.334.

MARINHO, C. L. F; RIBEIRO, L. S.. Inatividade física e surgimento de patologias em adolescentes: uma revisão bibliográfica. Revista Uningá, [S.l.], v. 56, n. 1, p. 108-113, mar. 2019. ISSN 2318-0579.

MORAIS, N. S. Tendência secular do estado nutricional, capacidade preditiva e pontos de corte de índices antropométricos para identificar adiposidade corporal e risco cardiometabólico em adolescentes. 2019. 140 folhas. Dissertação para obtenção de título de Mestre em Ciência da Nutrição - Universidade Federal de Viçosa, Viçosa/MG, 2019.

MATJUDA, E. N. et al. Association of Hypertension and Obesity with Risk Factors of Cardiovascular Diseases in Children Aged 6-9 Years Old in the Eastern Cape Province of South Africa. Children, v. 7, n. 4, p. 25, 2020. https://doi.org/10.3390/children7040025.

PRÉCOMA, D. B. et al. Atualização da Diretriz de Prevenção Cardiovascular da Sociedade Brasileira de Cardiologia - 2019. Arquivos Brasileiros de Cardiologia. 2019; [online]. ahead print, PP.0-0. https://doi.org/10.5935/abc.20190204.

RABELO, M. O. et al. O mutirão de prevenção ao câncer: um relato de experiência no âmbito da extensão universitária. Revista Intercâmbio, v. 7, p. 406-413, 2016.

RODRIGUES, E. S. R. et al. Fatores de risco para doenças ateroscleróticas cardiovasculares em escolares: uma ação preventiva primária. Relato de experiência. Amazônia: Science \& Health, v. 4, n. 2, p. 16-26, 2016.

SALAZAR RENDÓN, J. C.; MÉNDEZ, N.; AZCORRA, H. Association between overweight and obesity with school performance in secondary students in Merida, México. Bol Med Hosp Infant Mex, v. 75, n. 2, p. 94-103, 2018. https://doi.org/10.24875/BMHIME.M18000028.

SANTOS, J. B.; SERRA, H.; PEREIRA, A. S. Compreensão de estudantes do ensino médio sobre sedentarismo, nível de atividade física e exposição a comportamentos sedentários. Revista Thema, v. 16, n. 4, p. 818-831, jan. 2020. https://doi.org/10.15536/thema.V16.2019.818831.1118 . 
Ação extensionista de promoção da saúde e prevenção de doenças cardiovasculares para alunos de uma escola da Bahia

SOCIEDADE BRASILEIRA DE DIABETES. Diretrizes da sociedade brasileira de diabetes: 2019-2020. Diretrizes SBD, 2019.

SILVA, G. S. F. et al. Avaliação do nível de atividade física de estudantes de graduação das áreas saúde/biológica. Revista Brasileira de Medicina do Esporte, n.13, v.1, p.39-42, 2007. https://doi.org/10.1590/S1517-86922007000100009.

Recebido em: 31/07/2020

Aceito em: 11/08/2021 